\title{
Le travail à temps partiel durant les études chez les élèves du secondaire
}

Impacts sur leur adaptation scolaire et psychosociale

Students Working Part-Time While Attending High School

Impacts on Scholastic and Psychosocial Adaptation

El trabajo a tiempo parcial durante los estudios entre los

alumnos de secundaria

Impactos solore la adaptación escolar y psicosocial

\section{Michelle Dumont}

Volume 35, numéro 1, printemps 2007

L'insertion socioprofessionnelle des jeunes

URI : https://id.erudit.org/iderudit/1077960ar

DOI : https://doi.org/10.7202/1077960ar

\section{Aller au sommaire du numéro}

\section{Éditeur(s)}

Association canadienne d'éducation de langue française

\section{ISSN}

0849-1089 (imprimé)

1916-8659 (numérique)

\section{Découvrir la revue}

\section{Citer cet article}

Dumont, M. (2007). Le travail à temps partiel durant les études chez les élèves du secondaire : impacts sur leur adaptation scolaire et psychosociale. Éducation et francophonie, 35(1), 161-181. https://doi.org/10.7202/1077960ar

\section{Résumé de l'article}

Le travail à temps partiel (TTP) des adolescentes et adolescents durant les études est un phénomène social de plus en plus répandu en Amérique du Nord. Pourtant, que savons-nous de ses impacts, à court et à moyen termes, sur l'adaptation scolaire et psychosociale des élèves entre le milieu et la fin du secondaire? Dans le cadre d'une étude québécoise, des questionnaires standardisés ont été remplis par des élèves en troisième, quatrième et cinquième secondaire : tracas quotidiens (Plancherel et al., 1997), stratégies adaptatives (Frydenberg et al., 1993), problèmes psychopathologiques (Achenbach, 1991), estime de soi (Rosenberg, 1965), optimisme (Scheier et al., 1985), autonomie (Greenberger et al., 1974) et travail à temps partiel (adaptation du MÉQ, 1994). Les relevés de notes des élèves en mathématiques, en français ainsi que le relevé général furent également recueillis.

Globalement, les résultats révèlent que le TTP durant les études s'avère un facteur de risque pour l'adaptation scolaire des élèves en troisième et en quatrième secondaire travaillant plus de onze heures par semaine, ce qui n'est pas le cas de ceux appartenant à la même catégorie en cinquième secondaire. Par contre, aucun coût réel associé au TTP durant les études n’a été observé sur l'adaptation psychosociale des adolescentes et adolescents et cela, aux trois cycles scolaires. D'autres analyses indiquent qu'un TTP effectué en troisième secondaire ne permet pas de prédire la sévérité des tracas quotidiens, les ressources personnelles, les résultats scolaires et les comportements intériorisés et extériorisés mesurés en quatrième secondaire, exception faite des stratégies adaptatives productives et sociales évaluées en cinquième secondaire. La conclusion porte sur l'importance de renforcer les facteurs de protection de chaque élève afin de mieux faire face au tandem travail-études.

Tous droits réservés $@$ Association canadienne d’éducation de langue française, Ce document est protégé par la loi sur le droit d’auteur. L’utilisation des 2007 services d’Érudit (y compris la reproduction) est assujettie à sa politique d'utilisation que vous pouvez consulter en ligne.

https://apropos.erudit.org/fr/usagers/politique-dutilisation/ 


\title{
Le travail à temps partiel durant les études chez les élèves du secondaire : impacts sur leur adaptation scolaire et psychosociale
}

\author{
Michelle DUMONT \\ Université du Québec à Trois-Rivières, Québec, Canada
}

\section{RÉSUMÉ}

Le travail à temps partiel (TTP) des adolescentes et adolescents durant les études est un phénomène social de plus en plus répandu en Amérique du Nord. Pourtant, que savons-nous de ses impacts, à court et à moyen termes, sur l'adaptation scolaire et psychosociale des élèves entre le milieu et la fin du secondaire? Dans le cadre d'une étude québécoise, des questionnaires standardisés ont été remplis par des élèves en troisième, quatrième et cinquième secondaire: tracas quotidiens (Plancherel et al., 1997), stratégies adaptatives (Frydenberg et al., 1993), problèmes psychopathologiques (Achenbach, 1991), estime de soi (Rosenberg, 1965), optimisme (Scheier et al., 1985), autonomie (Greenberger et al., 1974) et travail à temps partiel (adaptation du MÉQ, 1994). Les relevés de notes des élèves en mathématiques, en français ainsi que le relevé général furent également recueillis. Globalement, les résultats révèlent que le TTP durant les études s'avère un facteur de risque pour l'adaptation scolaire des élèves en troisième et en quatrième secondaire travaillant plus de onze heures par semaine, ce qui n'est pas le cas de ceux appartenant à la même catégorie en cinquième secondaire. Par contre, aucun coût réel associé au TTP durant les études n'a été observé sur l'adaptation psychosociale des 
adolescentes et adolescents et cela, aux trois cycles scolaires. D'autres analyses indiquent qu'un TTP effectué en troisième secondaire ne permet pas de prédire la sévérité des tracas quotidiens, les ressources personnelles, les résultats scolaires et les comportements intériorisés et extériorisés mesurés en quatrième secondaire, exception faite des stratégies adaptatives productives et sociales évaluées en cinquième secondaire. La conclusion porte sur l'importance de renforcer les facteurs de protection de chaque élève afin de mieux faire face au tandem travail-études.

\section{ABSTRACT}

\section{Students Working Part-Time While Attending High School : Impacts on Scholastic and Psychosocial Adaptation}

Michelle DUMONT, University of Quebec in Trois-Rivières, Quebec, Canada

Part-time employment (PTE) among adolescents during their studies is an increasingly common social phenomenon in North America. However, how much do we know about its short and long term impacts on students' scholastic and psychosocial adjustment between the middle and end of their secondary school studies? In the framework of a Québec study, standardized questionnaires were completed by students in the third, fourth and fifth years of secondary school in terms of daily problems, (Plancherel et al., 1997), adaptive strategies (Frydenberg et al., 1993), psychopathological problems (Achenbach, 1991), self esteem (Rosenberg, 1965), optimism (Scheier et al., 1985), autonomy (Greenberger et al., 1974) and part-time work (The Québec ministry of education's adaptation (MÉQ), 1994). The students' math and French marks, as well as their overall results were also collected. Globally, the results reveal that PTE while attending school is a risk factor for school adjustment for students in secondary three and four working more than eleven hours per week, but not for those in the same category in secondary 5 . However, no real costs associated to PTE were observed on the psychosocial adjustment of adolescents in any of the three school cycles. Other analyses indicate that having a part time job in secondary three does not allow a prediction to be made about the severity of daily problems, personal resources, marks, and interiorized and exteriorized behaviours measured in secondary four, with the exception of adaptive productive and social strategies evaluated in secondary five. The conclusion emphasizes the importance of strengthening each student's protection factors so they can to deal more effectively with the workstudy combination. 


\section{RESUMEN}

\section{El trabajo a tiempo parcial durante los estudios entre los alumnos de secundaria : impactos sobre la adaptación escolar y psicosocial}

Michelle DUMONT

Universidad de Québec en Trois-Rivières, Québec, Canadá

El trabajo a tiempo parcial (TTP) de los adolescentes durante los estudios es un fenómeno social cada vez más común en América del Norte. ¿̇in embargo, qué sabemos de sus impactos, a corto y mediano plazo, sobre la adaptación escolar y psicosocial de los alumnos a la mitad o al final de la secundaria? En el marco de un estudio quebequence, se aplicaron cuestionarios estandardizados entre los alumnos de tercero, cuarto y quinto de secundaria: preocupaciones cotidianas (Plancherel et al, 1997), estrategias de adaptación (Frydenberg et al., 1993), problemas psicopatológicos (Achenbach, 1991), auto-estima (Rosenberg, 1965), optimismo (Scheier et al., 1985), autonomía (Greenberger et al., 1974) y trabajo a tiempo parcial (adaptación del MEQ, 1994). Las calificaciones en matemáticas, en francés y en las otras materias fueron asimismo recopiladas. Globalmente, los resultados muestran que el TTP durante los estudios se presenta como un factor de riesgo para la adaptación escolar de los alumnos de tercero y cuarto de secundaria que trabajan más de once horas por semana, pero no para los alumnos de quinto de secundaria. En cambio, ningún costo real asociado al TTP durante los estudios ha sido observado sobre la adaptación psicosocial de los adolescentes y esto, para los tres ciclos escolares. Otros análisis indican que un TTP durante el tercero de secundaria no permite predecir la severidad de las preocupaciones cotidianas, los recursos personales, los resultados escolares y los comportamientos interiores y exteriores medidos en cuarto de secundaria, excepción hecha de las estrategias de adaptación productivas y sociales evaluadas en quinto de secundaria. Las conclusiones subrayan la importancia de reforzar los factores de protección de cada alumno para bien confrontar el tándem trabajo-estudios.

\section{Introduction}

L'adolescence est marquée par des changements nombreux, intenses et rapides sur le plan pubertaire, identitaire et relationnel, sans parler des attentes de performance. Or, une des tâches développementales associées à cette période de la vie concerne la construction de l'identité. Le fait de travailler à temps partiel peut forcer ce processus, car dans de nouveaux contextes environnementaux, l'adolescent apprendra à mieux se connaître, à identifier de quoi il est capable, ce qu'il veut et ce qu'il peut faire de sa vie. Or, dans la société québécoise, on note une acceptation 
croissante des adultes à l'égard du travail à temps partiel des adolescentes et adolescents. En effet, il n'est pas rare d'entendre dire "cela va forger son caractère et développer son sens des responsabilités ». Parallèlement à ce changement de mentalité, la société de consommation dans laquelle les adolescentes et adolescents évoluent favorise l'accès rapide au monde du travail afin de se procurer certains biens jugés nécessaires pour les uns et inutiles pour les autres. Dans une étude menée par Dumont (2000), ne pas avoir assez d'argent de poche constitue un tracas quotidien formulé par les deux tiers des élèves de cinquième secondaire. Par conséquent, il n'est pas étonnant de constater que le tandem travail-études fait de plus en plus partie de la réalité nord-américaine des adolescentes et adolescents des années 2000.

Devant ces constats, on note, depuis plus de vingt ans, un intérêt pour l'évaluation des impacts liés au travail à temps partiel (TTP) durant les études sur le rendement scolaire et le bien-être psychologique des adolescentes et adolescents. Malgré cet intérêt scientifique émergent, les recherches québécoises sont peu nombreuses et la question des coûts et bénéfices reste sans réponse définitive. Par conséquent, cette étude identifie les coûts et bénéfices, à plus ou moins long terme, reliés au tra-

Un des éléments novateurs de cette étude consiste à évaluer les impacts liés à l'exercice d'un travail à temps partiel durant les études sur la sévérité des tracas quotidiens, sur les performances scolaires, sur les comportements intériorisés et extériorisés, ainsi que sur certaines ressources personnelles. vail à temps partiel durant l'année scolaire. Un des éléments novateurs de cette étude consiste à évaluer les impacts liés à l'exercice d'un travail à temps partiel durant les études sur la sévérité des tracas quotidiens, sur les performances scolaires, sur les comportements intériorisés et extériorisés, ainsi que sur certaines ressources personnelles, variables souvent négligées dans la documentation, et cela, auprès de cinq groupes de travailleuses et de travailleurs mesurés entre le milieu et la fin du secondaire (groupes: ne travaille pas, travaille entre 1 à 5 heures par semaine, travaille entre 6 à 10 heures par semaine, travaille plus de 11 heures par semaine). Un autre élément de nouveauté consiste à évaluer la valeur prédictive des conséquences à plus ou moins long terme du TTP en troisième secondaire sur l'adaptation scolaire et psychosociale des élèves en quatrième et en cinquième secondaire (tracas quotidiens, ressources personnelles, performances scolaires et détresse psychologique). L'évaluation des conséquences liées au TTP durant les études sur une période de trois ans a rarement été rapportée dans la documentation.

\section{Loi sur les normes du travail}

Comme entrée en matière, soulignons les droits et obligations des employeurs à l'égard des adolescentes et adolescents afin d'assurer leur sécurité et leur plein développement. Globalement, la Loi sur les normes du travail interdit à un employeur: 1) de faire effectuer par un enfant un travail disproportionné à ses capacités ou susceptible de porter atteinte à son éducation, à sa santé ou à son développement, 2) de faire travailler un enfant de moins de 14 ans sans le consentement écrit du parent, 3) de faire travailler, durant les heures de classe, un enfant tenu de fréquenter l'école, 4) de faire travailler, entre 23 heures et 6 heures le lendemain, un enfant tenu de fréquenter l'école, sauf si l'enfant livre des journaux, ou s'il effectue un travail à titre de créateur ou d'interprète dans certains domaines de production artistique (Commission des normes du travail, 2003; Dumont, 2005). 


\section{Conséquences liées au travail à temps partiel des adolescentes et adolescents durant leurs études}

À quel moment peut-on dire que le travail à temps partiel durant les études constitue un facteur de risque ou de protection pour l'adaptation scolaire et psychosociale des adolescentes et adolescents? Les résultats souvent contradictoires observés dans la documentation soutiennent à la fois la thèse des coûts, et des bénéfices. Les répercussions négatives associées au travail à temps partiel sont importantes, surtout si certains seuils critiques sont franchis soit 15 heures par semaine consacrées à un emploi durant les études, mais surtout à partir de 20 heures par semaine. Rappelons que l'adolescence est une période marquée par d'importants

Les élèves qui cumulent le double rôle travail-études augmentent ainsi le risque de présenter des problèmes scolaires et psychosociaux sous le poids de la fréquence et de la sévérité des facteurs de stress et des exigences liées à ces deux fonctions. changements de maturation physique, identitaire, intellectuelle et socioaffective. Les élèves qui cumulent le double rôle travail-études augmentent ainsi le risque de présenter des problèmes scolaires et psychosociaux sous le poids de la fréquence et de la sévérité des facteurs de stress et des exigences liées à ces deux fonctions.

\section{Coûts}

Globalement, un travail à temps partiel intense serait associé sur le plan de l'école à des aspirations scolaires moins élevées, à de la fatigue au point de s'endormir sur son pupitre (Bachman et Schulenberg, 1993; Beauchesne et Dumas, 1993; Largie, Field, Hernandez-Reif, Sanders et Diego, 2001), à une baisse de rendement scolaire et d'engagement scolaire (Steinberg \& Dornbusch, 1991) et à une probabilité de décrochage scolaire (D’Amico, 1984; Santana, Cooper, Roberts et Araújo-Filho, 2005). Si un lien peut être établi entre le TTP durant les études et le décrochage scolaire, ce dernier peut aussi résulter d'un manque d'intérêt pour l'école et d'un retard dans le cheminement scolaire. Ces types de problèmes peuvent survenir bien avant l'occupation d'un emploi à temps partiel ou alors découler de facteurs externes à l'école comme par exemple un échec amoureux, le décès d'un être cher ou une alternative à la pauvreté. Sur le plan de la santé, le TTP serait associé à une baisse d'activités sportives, à une réduction des heures consacrées au sommeil, à une insatisfaction liée au temps accordé aux loisirs (Bachman et al., 1993), à une augmentation de la consommation de substances et/ou d'alcool (Kouvonen et Lintonen, 2002ab; Largie et al., 2001), à une baisse d'activités sportives et parascolaires (Safron, Schulenberg et Bachman, 2001). Sur le plan personnel, familial et social, le TTP pourrait mener à des comportements agressifs, à des disputes avec les parents, à des troubles avec la police (Bachman et al., 1993), à des symptômes dépressifs et à un appauvrissement de la relation parents-adolescent: peu de conversations et de piètre qualité, moins de temps passé ensemble et de contacts physiques (Largie et al., 2001).

\section{Bénéfices}

En contrepartie, Green (1990) suggère que le stéréotype négatif lié au travail à temps partiel est peu justifié puisque, selon lui, il facilite la transition entre la fin du secondaire et l'entrée sur le marché du travail, en plus d'offrir des expériences sociales complémentaires à l'environnement familial ou scolaire. Malgré ce constat, il suggère que certains jeunes croient que le travail à temps partiel interfère avec 
d'autres intérêts personnels. Parmi les avantages liés au travail à temps partiel, Mortimer, Harley et Staff (2002) rapportent, selon un relevé de la documentation, le développement du sens des responsabilités, de la confiance en soi, de la gestion efficace du temps (ponctualité) et de l'estime dans le regard des pairs. Selon les adolescentes et adolescents eux-mêmes, leur emploi les aide à participer aux discussions en classe et à reconnaître l'importance de l'éducation si on veut éviter d'occuper un emploi répétitif, monotone et peu payant le reste de sa vie. Sans pourtant reposer sur une évaluation empirique de la question, on peut penser que d'autres gains peuvent découler d'une incursion précoce dans le monde du travail. Notons entre autres l'expérience acquise dans un monde d'adultes et l'effet structurant que le travail à temps partiel peut apporter sur l'arbitrage entre le temps d'étude et le temps de travail. Le sentiment d'être utile et apprécié pourrait aussi compter parmi les retombées positives du travail à temps partiel et cela surtout si l'élève échoue à l'école ou est mis à l'écart pour des raisons d'intimidation ou de discrimination. De plus, la participation au marché du travail peut favoriser le développement de compétences sociales et d'habiletés qui faciliteront l'insertion professionnelle, telles que la capacité à surmonter sa timidité, le fait d'apprendre à s'affirmer, à mieux se connaître, à être responsable et ponctuel, à prendre des initiatives, à respecter la valeur du travail, de l'effort et de l'argent, à gérer son temps, à collaborer, à travailler avec des appareils sophistiqués, faire l'apprentissage d'une langue seconde, etc. L'exercice d'un travail à temps partiel modéré peut s'avérer une alternative de réussite, mais pas la seule, pour les élèves qui s'adaptent mal au monde scolaire et dont la persévérance est en péril. Enfin, le TTP rend également la transition entre le monde scolaire des adolescentes et adolescents et l'univers professionnel des adultes plus souple et moins anxiogène pour ces élèves en comparaison avec ceux qui auraient été privés d'une telle expérience plus tôt dans le développement. Mortimer, Harley et Staff (2002) concluent que le bien-être personnel de l'adolescent se voit amélioré quand il se dit satisfait de son salaire, qu'il peut sortir plus souvent avec ses amis et dépenser son argent librement. Somme toute, une insertion professionnelle précoce peut être vue comme une excellente source de socialisation facilitant la transition entre la fin du secondaire et le début de l'intégration de l'élève au monde du travail.

\section{Détresse psychologique et ressources personnelles comme facteurs associés au travail à temps partiel des adolescentes et adolescents durant les études}

Puisque la question du rapport coûts et bénéfices lié au travail à temps partiel est loin d'être résolue, nous en évaluerons les liens avec des ressources personnelles et adaptatives qui jusqu'ici ont peu été étudiées dans ce type d'études.

\section{Détresse psychologique : une mauvaise adaptation aux facteurs de stress}

Occuper un double rôle travail-études peut mettre l'élève sous pression devant les exigences nouvelles et nombreuses qu'offrent ces différents milieux de socialisation, ce qui par ricochet risque d'affecter son bien-être psychologique. Or, plusieurs recherches ont démontré un lien entre le stress et un faible rendement scolaire de 
même qu'entre le stress et des problèmes d'adaptation psychosociale (Dumont, Leclerc et Deslandes, 2003), dont les troubles intériorisés (Steinhausen et Winkler Metzke, 2001), les symptômes somatiques, anxieux et dépressifs, le retrait social, les comportements d'agressivité et de délinquance (Reynolds, O'Koon, Papademetriou, Szczygiel et Grant, 2001) et le risque suicidaire (Leclerc, Pronovost et Dumont, 2004). De plus, sous l'influence du stress, un adolescent peut voir augmenter sa consommation de substances nocives pour sa santé surtout s'il travaille de nombreuses heures par semaine et s'il possède de l'argent pour s'en procurer. Or, il faut savoir que la consommation à risque serait également associée à un désir d'abandon scolaire ou à une diminution des résultats scolaires (Guyon et Desjardins, 2002).

\section{Ressources personnelles}

Outre les conséquences néfastes associées à la pratique d'un travail à temps partiel comme source additionnelle de stress pouvant affecter la santé psychologique des jeunes, plusieurs résultats de recherche suggèrent une diminution de ces impacts lorsqu'il y a présence et utilisation de ressources personnelles dites positives. Un certain nombre de variables jugées protectrices pour l'adaptation des adolescentes et adolescents seront évaluées ici en lien avec la pratique d'un TTP durant les études.

Stratégies d'adaptation (“coping”): Adopter de mauvaises stratégies adaptatives en réponse à des situations de stress, comme le fait d'assumer le double rôle travailétudes, représente un coût pour l'adaptation. En effet, les adolescentes et adolescents vivant un niveau de stress chronique élevé montrent plus d'anxiété et de dépression, s'engagent davantage dans des comportements antisociaux et rapportent moins de coping actif (Schmeelk-Cone et Zimmerman, 2003). Le coping évitant ou non productif serait quant à lui associé aux troubles intériorisés ou extériorisés (Dumont, Leclerc et Pronovost, 2000; Seiffge-Krenke, 2000; Steinhausen et Metzke, 2001), à l'anxiété exprimée à l'égard des stresseurs familiaux, scolaires, avec les pairs (Griffith, Dubow et Ippolito, 2000) et au risque suicidaire (Pronovost, Leclerc et Dumont, 2003).

Estime de soi: L'estime de soi est susceptible d'augmenter sous l'influence d'un travail à temps partiel valorisant. En revanche, une faible estime de soi serait associée à de moins bons résultats scolaires, à davantage de troubles intériorisés et extériorisés (DuBois, Bull, Sherman et Roberts, 1998), au coping évitant (Dumont et al., 1999), à un nombre plus élevé d'événements de vie (Balwin et Hoffmann, 2002), à plus de stress, de dépression, de psychosomatisation, de solitude et d'inquiétude exprimée face aux examens (Dumont et al., 2003).

Optimisme: On peut penser que la personnalité optimiste peut jouer un rôle médiateur dans la manière de bien répondre à des sources de stress comme celui relié au TTP durant les études. Par rapport aux personnes pessimistes, les optimistes rapportent un niveau d'estime de soi plus élevé, expérimentent moins de détresse psychologique (Creed, Patton et Bartrum, 2002), manifestent plus de joie et de bienêtre (Ben-Zur, 2003), optent davantage pour des stratégies adaptatives de type résolution de problèmes, mettent l'accent sur le côté positif des situations stressantes 
(Scheier, Kumari-Weintraub et Carver, 1986) et adoptent plus souvent un coping proactif (Greenglass, 2002).

Autonomie: À l'adolescence, on s'attend à davantage d'autonomie et de prise de responsabilité de la part des jeunes. Occuper un travail à temps partiel peut s'avérer un atout pour le développement de cet attribut personnel. Globalement, l'autonomie réfère à l'autodétermination de sa pensée, de ses sentiments, de ses comportements, ce qui soutient les concepts d'identité, d'indépendance, de compétence interpersonnelle et de responsabilité à assumer (Deslandes, Potvin et Leclerc, 1999). Cette caractéristique de la personnalité est associée au profil de la personne dite résiliente. Soulignons ici que ce domaine de recherche a été peu étudié en relation avec les variables scolaires et mérite qu'on s'y attarde davantage en particulier lorsqu'il s'agit de le relier au travail des jeunes comme accélérant du développement de cet attribut.

À la lumière de cette recension des écrits, on peut difficilement conclure que le travail à temps partiel agit comme facteur de protection ou de risque pour l'adaptation scolaire et psychosociale des adolescentes et adolescents. La contradiction dans les résultats de recherche proviendrait en partie des différences d'instruments de mesure, de la variabilité dans la taille des échantillons (entre 200 élèves et plusieurs milliers d'élèves) et dans les devis de recherche (longitudinaux par rapport à transversaux). Principalement, trois types de variables ont été étudiées dans ces recherches: 1) les unes mettent en relation certains indices scolaires et psychosociaux avec le nombre d'heures qui fut consacré à un travail à temps partiel durant

Nous formulons I'hypothèse que le nombre d'heures consacré à un travail à temps partiel en troisième secondaire influencera négativement le profil scolaire et psychosocial des élèves en quatrième et en cinquième secondaire. les études sans vraiment tenir compte des impacts à plus ou moins long terme, 2) les autres effectuent une comparaison intergroupes entre le statut de travailleur et celui de non-travailleur alors que 3) les dernières font porter leur objet d'étude sur l'évaluation des conditions de travail comme la sécurité notamment.

La présente étude s'inspire des deux premières stratégies de recherche en mettant en relation le nombre d'heures consacré à un travail à temps partiel durant les études avec une série de marqueurs liés aux ressources personnelles (stratégies adaptatives, optimisme, autonomie, estime de soi), aux tracas quotidiens et à l'adaptation psychosociale de type intériorisé et extériorisé. Cette mise en relation sera réalisée auprès de quatre groupes de travailleurs : ne travaille pas, travaille entre 1 et 5 heures par semaine, travaille entre 6 et 10 heures par semaine, travaille plus de 11 heures par semaine. À l'instar des recherches préalablement citées, nous formulons l'hypothèse que les élèves qui travaillent plus de 11 heures par semaine présenteront un profil scolaire et psychosocial déficitaire par rapport aux trois autres groupes de comparaison (ceux qui ne travaillent pas, ceux qui travaillent entre 1 à 6 heures par semaine et ceux qui travaillent entre 6 et 10 heures par semaine). Puis, il sera question d'évaluer la valeur prédictive du travail à temps partiel exercé en troisième secondaire sur la sévérité des tracas quotidiens, sur les ressources personnelles et sur l'adaptation scolaire et psychosociale mesurées en quatrième ou en cinquième secondaire. Nous formulons l'hypothèse que le nombre d'heures consacré à un travail à temps partiel en troisième secondaire influencera négativement le profil scolaire et psychosocial des élèves en quatrième et en cinquième secondaire. 


\section{Méthode}

\section{Participantes et participants}

Une école publique issue de la région de la Mauricie a participé à cette étude québécoise. Le niveau socioéconomique familial est, en moyenne, de 52100 \$, selon l'enquête de l'Institut économique de Montréal de l'Institut Fraser (Actualité, 2003). Au total, 188 élèves en troisième secondaire ont participé à cette étude ( $N=106$ filles; $N=82$ garçons) ainsi que 171 élèves en quatrième secondaire ( $N=109$ filles; $N=62$ garçons) et 144 élèves en cinquième secondaire ( $N=97$ filles; $N=47$ garçons). Le taux de participation peu élevé s'explique en partie par le fait que l'autorisation écrite des parents était exigée. L'âge moyen est de 14,31 ans (é.t. $=0,596$ ) en troisième secondaire, de 15,34 ans (é.t. $=0,634$ ) en quatrième secondaire et de 16,31 ans (é.t. $=0,583$ ) en cinquième secondaire.

La présentation du projet aux élèves a été effectuée en classe par l'équipe de recherche composée d'étudiants gradués en psychologie inscrits en deuxième et troisième cycles. La passation collective des questionnaires a eu lieu au cours de deux périodes de 60 minutes chacune réparties sur deux semaines consécutives. Les collectes de données annuelles ont été réalisées à l'automne à la suite d'une première série d'examens scolaires. Les notes en mathématiques et en français ainsi que le relevé général ont été recueillis de façon concomitante avec la collecte des questionnaires. Les consentements des parents et des élèves ont été recueillis deux semaines avant la passation collective des questionnaires. Un tirage au montant de 10 \$ été offert dans chacune des classes et ceci à chaque année où se déroulait une collecte des données.

\section{Questionnaires}

Travail à temps partiel: Le TTP a été évalué à partir de l'adaptation du questionnaire de Beauchesne et Dumas (1993) et de l'entrevue semi-structurée proposée par Green (1990). Cette version adaptée fut testée auprès de 40 élèves en troisième, quatrième et cinquième secondaire travaillant peu (moins de 5 heures), moyennement (entre 6 et 14 heures) et beaucoup (plus de 15 heures) afin d'élaborer la version définitive du questionnaire utilisée dans la présente étude.

Estime de soi (Self-Esteem Scale; Rosenberg, 1965) : Ce questionnaire comporte 10 items qui évaluent le sentiment de valeur personnelle sur une échelle Likert à quatre points. Certains items doivent être recodés subséquemment. Dans notre étude, les alphas de Cronbach pour les trois niveaux scolaires varient entre 0,87 et 0,89 .

Stratégies d'adaptation des adolescents (Adolescent Coping Scale; Frydenberg et Lewis, 1993) : Cet inventaire de stratégies adaptatives (79 items) mesure la façon habituelle dont une adolescente ou un adolescent réagit aux problèmes de la vie courante (échelle Likert en cinq points). Le regroupement d'items permet de dégager 18 types de stratégies (chaque stratégie contenant de trois à cinq items) qui, à leur tour, définissent trois styles adaptatifs nommés productif (20 items), référence aux autres (18 items) et non productif (35 items). Dans notre étude, les alphas de 
Cronbach aux trois niveaux scolaires varient entre 0,83 et 0,87 pour le coping productif, entre 0,87 et 0,88 pour le coping référence aux autres et entre 0,90 et 0,92 pour le coping non productif.

Disposition à l'optimisme ou au pessimisme (LOT; Scheier et Carver, 1985) : Ce questionnaire comprend 12 items impliquant une échelle Likert en cinq points. Quatre items devront être recodés et quatre autres ne seront pas calculés dans le score final. Un haut score au LOT indique une tendance à l'optimisme. Dans notre étude, les alphas de Cronbach aux trois niveaux scolaires varient entre 0,81 et 0,86.

Échelle de mesure de l'autonomie - version D (Greenberger, Josselson, Knerr et Knerr, 1974; traduction et validation par Deslandes et al., 1999) : Ce questionnaire comprend 30 items distribués en trois sous-échelles, dont celle du score total d'autonomie (10 énoncés chacune impliquant une échelle de type Likert à quatre points). Dans notre étude, les alphas de Cronbach aux trois niveaux scolaires varient entre 0,90 et 0,92 .

Tracas quotidiens (Adolescent Hassles Inventory; Bobo, Gilchrist, Snow et Schinke, 1986; traduit et adapté par Plancherel, Bettschart, Bolognini, Dumont et Halfon, 1997): La version de Bobo et ses collaborateurs a été adaptée et validée auprès de préadolescents suisses et comprend, dans sa forme finale, 59 items (Plancherel et al., 1997). Ce questionnaire mesure la fréquence et le degré d'intensité des tracas quotidiens. Le sujet doit spécifier s'il a vécu ou non le problème et si oui, dans quelle mesure il en a été affecté durant les six derniers mois (échelle Likert variant de zéro à quatre points). Dans notre étude, les alphas de Cronbach aux trois niveaux scolaires varient entre 0,92 et 0,93 pour le score de sévérité aux tracas quotidiens.

Inventaire des problèmes de comportements du jeune âgé entre 11 et 18 ans (Youth Self Report; Achenbach, 1991): Le YSR, composé de 122 énoncés, évalue la perception qu'a l'adolescente ou l'adolescent de son profil internalisé et extériorisé (échelle Likert, 0 à 2). Ces syndromes sont déterminés selon des cotes T normalisées selon l'âge, le sexe et l'appartenance ou non à un groupe clinique. La fidélité testretest sur une semaine est, pour le score intériorisé, de 0,67 pour les 11-14 ans et de 0,91 pour les $15-18$ ans et, pour le score extériorisé, de 0,76 pour les plus jeunes et de 0,91 pour les plus vieux (Achenbach, 1991). Dans notre étude, les alphas de Cronbach aux trois niveaux scolaires varient entre 0,89 et 0,92 pour le trouble intériorisé et entre 0,82 et 0,86 pour le trouble extériorisé.

\section{Résultats}

Cette étude évalue les relations entre le travail à temps partiel et l'adaptation scolaire et psychosociale des adolescentes et adolescents. Dans un premier temps, des comparaisons intergroupes soulignent les similitudes et les différences d'adaptation scolaire et psychosociale aux trois cycles scolaires et cela pour trois groupes de travailleurs (travaille entre 1 à 5 heures, travaille entre 6 à 10 heures, travaille plus de 11 heures par semaine) et un groupe de non-travailleurs. Puis, d'autres analyses ont 
Le pourcentage d'élèves qui travaillent au-delà de 11 heures par semaine varie entre $15 \%$ environ (au milieu du secondaire) et $30 \%$ (à la fin du secondaire). aussi été effectuées afin de vérifier la valeur prédictive du travail à temps partiel exercé en troisième secondaire sur la sévérité des tracas quotidiens, sur les ressources personnelles et sur l'adaptation scolaire et psychosociale mesurées un ou deux ans plus tard, soit en quatrième et en cinquième secondaires.

D’entrée de jeu, soulignons qu'environ les deux tiers des élèves travaillent à temps partiel $(61,50 \%$ en troisième secondaire; 60,40\% en quatrième secondaire; $63,90 \%$ en cinquième secondaire). Les principaux emplois occupés en troisième et en quatrième secondaire sont: garder des enfants, distribuer des journaux, exercer un rôle d'animation. S'ajoutent à cela le service aux tables, la tenue d'une caisse, le travail dans un commerce ou dans un restaurant pour les élèves en cinquième secondaire. Le pourcentage d'élèves qui travaillent au-delà de 11 heures par semaine varie entre $15 \%$ environ (au milieu du secondaire) et $30 \%$ (à la fin du secondaire).

\section{Comparaison des différents groupes de travailleurs sur l'adaptation scolaire et psychosociale des adolescentes et adolescents}

Tel qu'attendu, le travail à temps partiel s'avère un facteur de risque pour l'adaptation scolaire des élèves qui travaillent plus de 11 heures par semaine. Comparativement à ceux qui travaillent peu (1 à 5 heures par semaine), les élèves qui travaillent beaucoup se disent plus fatigués et/ou plus stressés et ce, aux trois cycles scolaires ( fatigue» : $\chi 2(6, N=107)=14,427, \mathrm{p}=0,025$ en troisième secondaire; $\chi 2$ (6, $N=92)=22,434, \mathrm{p}=0,001$ en quatrième secondaire; $\chi 2(6, N=92)=23,364, \mathrm{p}=0,001$ en cinquième secondaire; "stress " : $\chi 2(6, N=107)=9,079, \mathrm{p}=0,169$ en troisième secondaire; $\chi 2(6, N=93)=14,118, \mathrm{p}=, 028$ en quatrième secondaire; $\chi 2(6, N=92)=$ $21,623, \mathrm{p}=0,001$ en cinquième secondaire). De plus, les élèves qui travaillent plus de 11 heures par semaine en troisième et en quatrième secondaire montrent des résultats scolaires statistiquement inférieurs aux autres groupes de comparaison (voir figures 1 et 2). Plus spécifiquement, pour les élèves en troisième secondaire, on peut noter une différence intergroupes au score obtenu en français $(F(3,167)=4,008$, $\mathrm{p}=0,009)$ ainsi que pour la moyenne générale $(F(3,170)=2,665, \mathrm{p}=0,050)$, mais pas pour la performance en mathématiques $(F(3,170)=2,290, \mathrm{p}=0,080)$. Plus spécifiquement, les élèves qui travaillent plus de 11 heures par semaine obtiennent une note en français statistiquement inférieure $(68,13 \%)$ à celle des élèves qui ne travaillent pas ( $\mathrm{p}=0,010 ; 76,55 \%)$, qui travaillent entre 1 à 5 heures $(\mathrm{p}=0,012 ; 77,02 \%)$ ou qui travaillent entre 6 et10 heures par semaine ( $p=0,020 ; 76,60 \%)$. Les plus gros travailleurs montrent aussi un relevé général inférieur (72,13\%) aux élèves qui ne travaillent pas ( $\mathrm{p}=0,043 ; 77,36 \%$ ). En ce qui concerne les élèves de quatrième secondaire, on peut relever une différence intergroupes pour la moyenne générale $(F(3,149)=2,962, \mathrm{p}=0,034)$, mais pas pour les mathématiques $(F(3,149)=1,325$, $\mathrm{p}=0,268)$, ni pour le français $(F(3,149)=1,801, \mathrm{p}=0,149)$. Plus spécifiquement, les élèves qui travaillent plus de 11 heures par semaine obtiennent un relevé de notes inférieur (73,04\%) aux élèves qui travaillent entre 1 à 5 heures par semaine $(p=0,027$; $79,03 \%)$. En revanche, aucune différence intergroupes sur le plan des performances scolaires n'a été observée en cinquième secondaire. Autrement dit, à la fin du secondaire, les quatre groupes de travailleurs ne se distinguent pas sur les notes en 
mathématiques $(\mathrm{F}(3,136)=1,056, \mathrm{p}=0,370)$, ni sur les notes en français $(F(3,136)$ $=1,958, \mathrm{p}=0,123)$, ni en ce qui a trait à la moyenne générale $(F(3,136)=0,146$, $\mathrm{p}=0,932$ ) (voir figure 3 ).

D'autres résultats indiquent que les trois cycles scolaires se distinguent également sur le plan des aspirations scolaires. En effet, le désir de poursuivre des études à des niveaux supérieurs chez les élèves qui consacrent beaucoup de temps à leur TTP est inférieur à celui des élèves qui travaillent moins ou qui ne travaillent pas du tout $\left(\chi 2(12, N=167)=22,734, \mathrm{p}=0,030\right.$ en troisième secondaire; $\chi^{2}(12, N=145)$ $=22,177, \mathrm{p}=0,040$ en quatrième secondaire). À titre illustratif, $30,4 \%$ des élèves qui travaillent plus de 11 heures par semaine en troisième secondaire souhaitent entreprendre des études universitaires comparativement à 75,8\% de ceux qui ne travaillent pas. Des résultats similaires ont été trouvés pour les élèves en quatrième secondaire (soit $52 \%$ versus $69 \%$ ). Contre toute attente, ce résultat n'a pas été observé en cinquième secondaire $(\chi 2(12, N=133)=5,957, \mathrm{p}=0,918)$. Autrement dit, la distribution des participantes et participants aux différentes catégories d'aspiration scolaire est statistiquement similaire entre les quatre groupes de comparaison (catégories: études collégiales générales, études collégiales techniques, études secondaires, diplôme d'études professionnelles, abandon avant la fin des études). Par exemple, les élèves qui travaillent beaucoup en cinquième secondaire souhaitent réaliser des études universitaires dans une proportion similaire aux trois groupes de comparaison (ne travaille pas $=57,4 \%$; travaille entre 1 à 5 heures $=68,8 \%$; travaille entre 6 à 10 heures $=55,2 \%$; travaille plus de 11 heures par semaine $=53,7 \%$ ).

Figure 1

Résultats scolaires selon le statut de travailleur en troisième secondaire (analyse univariée)

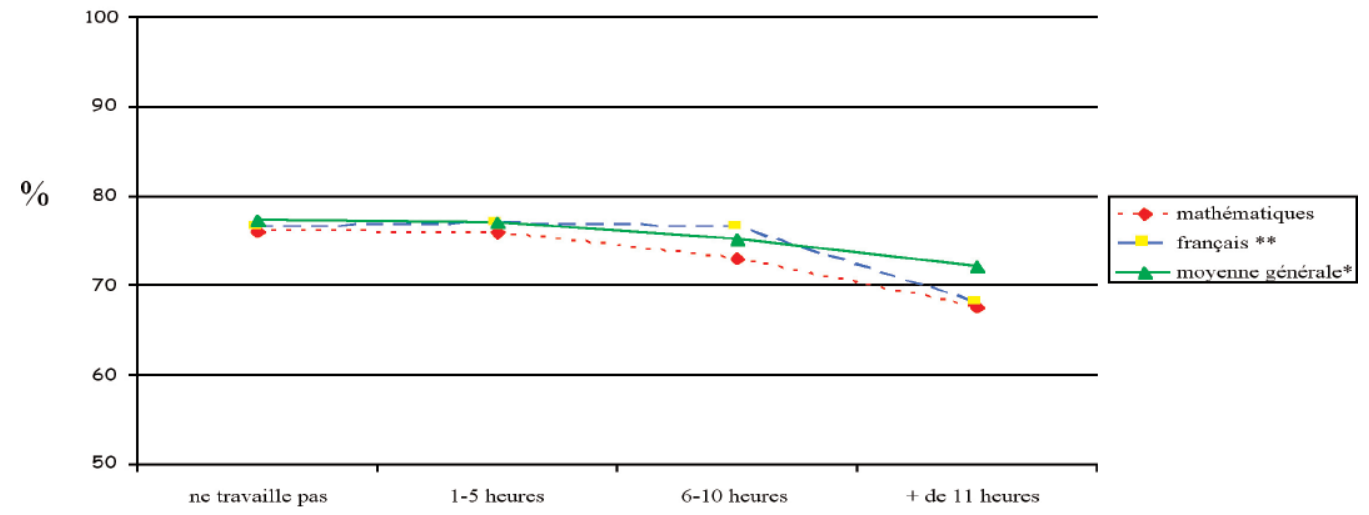

$* \mathrm{p}<0,05 ; * * \mathrm{p}<0,01$ 
Figure 2

Résultats scolaires selon le statut de travailleur en quatrième secondaire (analyse univariée)

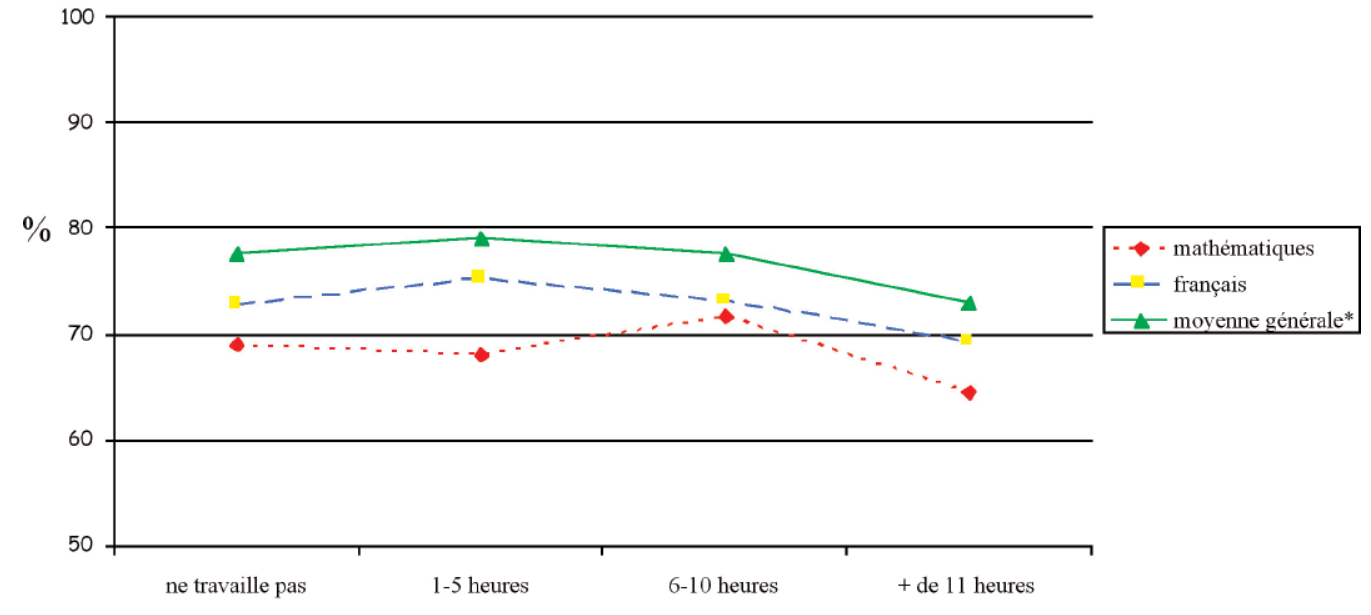

$* \mathrm{p}<0,05$

Figure 3

Résultats scolaires selon le statut de travailleur en cinquième secondaire (analyse univariée)

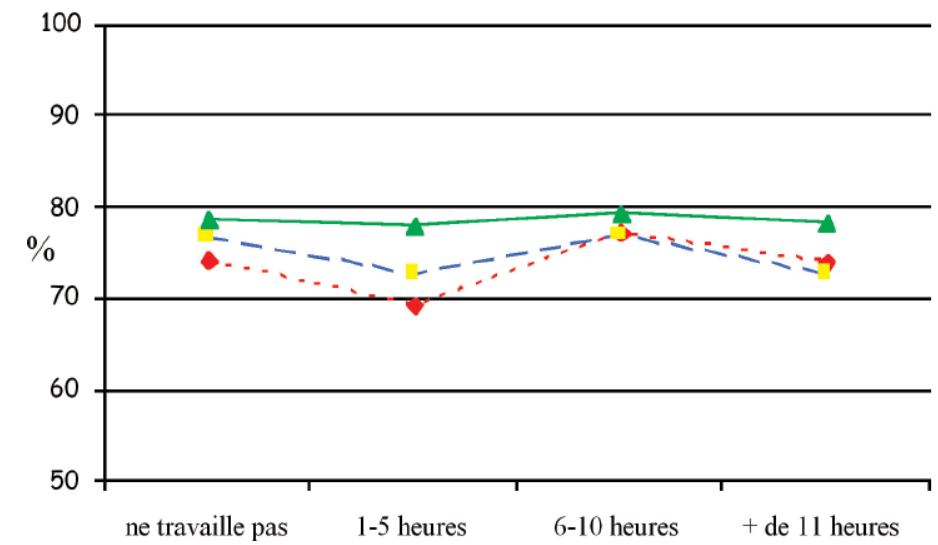

$$
\begin{aligned}
& -\downarrow \text { - mathématiques } \\
& -- \text { français } \\
& \longrightarrow \text { - moyenne générale }
\end{aligned}
$$


D'autres résultats issus d'analyses univariées indiquent que le travail à temps partiel ne représente pas un risque réel pour l'adaptation psychosociale des élèves et cela entre le milieu et la fin du secondaire. En effet, la comparaison entre le profil psychosocial des trois groupes de travailleurs (travaille entre 1 à 5 heures, travaille entre 6 et 10 heures, travaille plus de 11 heures) et celui des non-travailleurs ne révèle pas de différence statistiquement significative aux trois cycles scolaires. En effet, peu importe le groupe d'appartenance, les élèves en troisième et en quatrième secondaire adoptent relativement le même type de stratégies pour faire face aux difficultés de l'adolescence (styles productif, référence aux autres et non productif), ils obtiennent des scores similaires aux questionnaires d'estime de soi, d'autonomie et d'optimisme, ils expérimentent le même degré de sévérité des tracas quotidiens et de fréquence de symptômes intériorisés (retrait social, somatisation et anxiété) et extériorisés (délinquance et agressivité). Cependant, si en cinquième secondaire le profil psychosocial des non-travailleurs est relativement le même que celui des trois groupes de travailleurs, notons toutefois deux exceptions où les scores d'optimisme $(F(3,139)=3,376 ; \mathrm{p}=0,020)$ et d'autonomie $(F(3,140)=3,769, \mathrm{p}=0,012)$ se sont avérés plus élevés chez les élèves qui travaillent davantage d'heures par semaine. Plus spécifiquement, les élèves qui travaillent beaucoup à cet âge (plus de 11 heures par semaine) se disent plus optimistes $(p=0,030)$ et autonomes $(p=0,015)$ que ceux qui travaillent entre 1 à 5 heures par semaine.

\section{Conséquences à plus long terme du travail à temps partiel entre le milieu et la fin du secondaire}

Des analyses de régressions simples ont été réalisées afin d'évaluer les conséquences d'un travail à temps partiel exercé au milieu du secondaire sur l'adaptation psychosociale et scolaire mesurée un ou deux ans plus tard. Globalement, le nombre d'heures consacré à un travail à temps partiel en troisième secondaire ne permet pas de prédire, un an plus tard, soit en quatrième secondaire, la sévérité des tracas quotidiens, les ressources personnelles (l'estime de soi, les trois styles de coping, l'autonomie, l'optimisme), les notes scolaires (français, mathématiques, relevé général) et l'adaptation psychosociale (troubles intériorisés et extériorisés). Par contre, le nombre d'heures consacré à un TTP exercé en troisième secondaire prédirait, deux ans plus tard, soit en cinquième secondaire, l'utilisation plus fréquente des stratégies adaptatives productives $\left.C_{-}=0,21 ; \mathrm{p}=0,035\right)$ et de type référence aux autres $C_{-}=0,21$; $\mathrm{p}=0,036$ ) pour faire face aux difficultés de l'adolescence.

\section{Discussion}

Si la thèse des coûts ou des bénéfices liés au travail des adolescentes et adolescents ne fait pas encore consensus dans la documentation, tous s'entendent pour dire que les adolescentes et adolescents ont la responsabilité de ne rien faire qui puisse nuire à leur développement, à leur réussite scolaire et à l'obtention d'un diplôme de fin d'études secondaires. En effet, intervenants psychosociaux, agents 
Pour les élèves en troisième et en quatrième secondaire qui travaillent plus de 11 heures par semaine, le tandem travail-études s'avère être un facteur de risque pour le domaine scolaire (aspiration scolaire moindre, moyenne générale plus faible, fatigue ressentie plus élevée). d'éducation, parents et décideurs publics demeurent prudents en matière d'encouragement face au travail des adolescentes et adolescents. C'est à partir de 11 heures (dans notre étude) et de 15 à 20 heures par semaine (dans la documentation) que des effets néfastes dus au travail à temps partiel durant les études se font surtout sentir sur l'adaptation scolaire et psychosociale des adolescentes et adolescents.

\section{Comparaison des différents groupes de travailleurs sur l'adaptation scolaire et psychosociale des adolescentes et adolescents}

Étant donné qu'il s'agit ici d'une seule école publique québécoise et que cette étude ne repose que sur des questionnaires auto-rapportés, la prudence s'impose quant à la généralisation des résultats à d'autres contextes scolaires ou culturels. Pour les élèves en troisième et en quatrième secondaire qui travaillent plus de 11 heures par semaine, le tandem travail-études s'avère être un facteur de risque pour le domaine scolaire (aspiration scolaire moindre, moyenne générale plus faible, fatigue ressentie plus élevée). Cependant, ce constat n'a pas été observé pour les élèves en cinquième secondaire. Cette absence d'effet négatif lié à l'exercice d'un travail à temps partiel intense sur l'adaptation scolaire des élèves en cinquième secondaire s'explique de diverses façons : ils ont fait des choix de carrière donnant un sens à leurs études, ils ont pris davantage conscience de l'importance des études afin d'éviter la précarité d'emploi, ils se sont habitués au double rôle travail-études et ont développé des stratégies efficaces pour ne pas compromettre leurs études, ils sélectionnent des cours moins exigeants, etc. À la lumière de ces résultats, un élève en troisième ou en quatrième secondaire qui présenterait des problèmes d'apprentissage et dont la motivation à l'école serait peu élevée devrait évaluer les coûts et bénéfices avant d'accepter un travail à temps partiel qui risquerait de compromettre sa réussite éducative. Enfin, notre première hypothèse de recherche confirme les conséquences négatives liées au double rôle travail-études assumé durant l'adolescence sur la réussite scolaire (Bachman et Schulenberg, 1993; Beauchesne et Dumas, 1993, 2001; Largie, Field, Hernandez-Reif, Sanders et Diego, 2001; Steinberg et Dornbusch, 1991).

$\mathrm{Si}$, dans notre recherche, l'adaptation scolaire est directement affectée par le travail à temps partiel durant les études chez les élèves en troisième et en quatrième secondaire, cela ne semble pas être le cas pour l'adaptation psychosociale, contrairement aux attentes de recherche (on observe, entre les quatre groupes de comparaison, l'usage des mêmes stratégies adaptatives pour faire face aux difficultés, des scores similaires aux questionnaires d'estime de soi, d'autonomie, d'optimisme, un degré de sévérité aux tracas quotidiens relativement similaire, des symptômes intériorisés et extériorisés d'une même ampleur). Même si les scores d'optimisme et d'autonomie sont plus élevés en quatrième et en cinquième secondaire chez les élèves qui travaillent plus de 11 heures par semaine, et cela par rapport aux élèves qui travaillent moins, les résultats ne permettent pas de dire si ces caractéristiques personnelles étaient plus élevées au départ chez ces élèves, ce qui les aurait incités à travailler plus d'heures par semaine, ou si c'est le résultat bénéfique de la pratique d'un TTP intense. Rappelons toutefois qu'un trait d'optimisme constitue un atout 
développemental pouvant atténuer le stress associé au double rôle école-travail compte tenu d'une attitude plus positive envers le changement et les sources de stress (Scheier et al., 1986). De plus, Mortimer et Staff (2004) suggèrent que le travail à temps partiel peut favoriser le développement de certaines ressources personnelles, ce qui, par ricochet, permettrait la construction d'une certaine forme de résilience. D'autres hypothèses explicatives peuvent être formulées afin de justifier l'absence de coût psychosocial observé aux trois cycles scolaires à la suite de l'exercice intense d'un travail à temps partiel durant les études. Par exemple, les élèves ne cumulent ce double rôle travail-études que depuis environ quatre mois, au moment de la collecte de données, et travaillent en moyenne moins de 10 heures par semaine. Ces élèves se situent donc sous le seuil critique du 15 heures par semaine plus observé dans la documentation alors que des conséquences néfastes pour l'adaptation psychosociale seraient observées sur des périodes plus longues de travail. Dans une étude future, il serait donc souhaitable de prendre des mesures au début et à la fin de l'année scolaire afin de tester l'hypothèse du temps et du nombre d'heures consacré à un TTP qui serait supérieur à 15.

\section{Conséquences à plus long terme du travail à temps partiel entre le milieu et la fin du secondaire}

Le nombre d'heures consacré à un travail à temps partiel au milieu de l'adolescence ne semble pas influencer de manière évidente le profil scolaire et psychosocial des élèves mesuré un ou deux ans plus tard, exception faite de la fréquence d'utilisation des stratégies adaptatives de type productif ou de type référence aux autres, évaluée en cinquième secondaire. Dans ce dernier cas de figure, il y aurait une association entre le fait de travailler à temps partiel au milieu de l'adolescence et une plus grande utilisation de stratégies adaptatives dites aidantes à la fin du secondaire. À la lumière de ces résultats, une analyse longitudinale de l'ensemble de ces variables serait de mise, car d'autres résultats issus de cette étude (Dumont, Leclerc, Massé et McKinnon, juillet 2006) laissent indiquer que le meilleur cheminement pour l'adaptation scolaire (augmentation du relevé scolaire général) correspond au profil d'élèves dont le nombre d'heures consacré à un travail à temps partiel durant les études diminue entre le milieu et la fin du secondaire. En revanche, les élèves dont le nombre d'heures consacré à un travail à temps partiel augmenterait entre la troisième et la cinquième secondaire verraient leurs notes en mathématiques diminuer et leurs conduites délictueuses augmenter. Malgré ceci, ils gagneraient en estime de soi et en autonomie, ce qui confirmerait l'hypothèse des gains associés au travail à temps partiel. D'autres bénéfices ont aussi été observés pour ceux qui travaillent beaucoup au milieu et à la fin du secondaire. Ils deviennent plus optimistes et adoptent un meilleur coping. 


\section{Conclusion}

Somme toute, ces résultats de recherche suggèrent une certaine prudence de la part des parents à encourager le travail à temps partiel des adolescentes et adolescents de troisième et de quatrième secondaire et cela, en ce qui a trait aux conséquences néfastes potentielles sur les performances et les aspirations scolaires. Toutefois, l'analyse des résultats dans la documentation ne fait que commencer et les nuances ne sont pas toutes établies. Par exemple, une exploration plus approfondie des différences individuelles (genre, âge, motivation et résultats scolaires, profil de personnalité, qualité de la relation parents-adolescent, type de style éducatif, valeurs personnelles reliées à l'école, culture de performance à l'école, etc.) permettrait d'expliquer en quoi le cumul travail-études ne pose pas de problèmes pour les élèves plus résistants au stress, alors qu'un nombre d'heures plus limité pourrait être recommandé à ceux qui s'avèrent plus vulnérables au stress.

L'impact du travail à temps partiel peut s'analyser sous l'angle des facteurs de protection dont chaque adolescente et adolescent dispose. Plus ces facteurs sont nombreux et utilisés, plus nous sommes en droit de penser que les risques scolaires et psychosociaux devraient diminuer. Ces ressources dites aidantes peuvent être de nature personnelle (gestion efficace du stress, niveau peu élevé d'anxiété, facilité à l'apprentissage et à l'étude, intérêt pour les études, aspiration scolaire élevée, but professionnel clair, sommeil récupérateur, santé, capacité d'organisation, implication sportive, etc.), familiale (parents qui encouragent, valorisent et s'impliquent dans la vie scolaire de leur enfant, qui aiment lire et résoudre des problèmes, qui entretiennent une relation parents-adolescent aimante et soutenante et qui encadrent les activités du jeune en matière de temps consacré au clavardage, aux passe-temps, aux activités sociales et sportives, au sommeil, aux études, etc.) et environnementale (faire partie d'un réseau d'amis studieux, aimant l'école et ayant des aspirations scolaires, disposer d'une structure scolaire qui favorise l'aide aux devoirs avec horaire flexible, évoluer dans un environnement scolaire encourageant l'excellence, etc.). À la lumière de cette étude, il appert important de renforcer le développement de ces facteurs de protection afin que l'élève puisse mieux faire face au tandem travail-études. Que l'adolescente ou l'adolescent travaille ou non pendant ses études, il n'en demeure pas moins qu'il a la responsabilité de prendre en charge ses apprentissages et de mettre en place des conditions favorisant son développement, son adaptation scolaire et son bien-être personnel et cela, grâce à la complicité parentale et au soutien constant de l'école.

\section{Remerciements}

Cette recherche a été subventionnée par le Conseil de recherches en sciences humaines du Canada et le Fonds d'aide financière du Décanat des études avancées et de la recherche de l'Université du Québec à Trois-Rivières. Un remerciement tout spécial est adressé aux élèves, aux enseignants, aux directeurs adjoints, à la direction de l'école, ainsi qu'aux assistants et professionnels de recherche qui ont rendu possible la réalisation de cette étude. 


\section{Références bibliographiques}

ACHENBACH, T. M. (1991). Manual for the Child Behavior Check-List 4-18 and 1991 Profile. Burlington: University of Vermont, 288 p.

ACTUALITÉ (21 novembre 2003). Palmarès 2003 des écoles secondaires, 133 p.

BACHMAN, J. G. et SCHULENBERG, J. (1993). How Part-Time Work Intensity Relates to Drug Use, Problem Behavior, Time Use, and Satisfaction Among High School Seniors : Are These Consequences or Merely Correlates? Developmental Psychology, vol. 29, nº 2, p. 220-235.

BALDWIN, S. A, et HOFFMANN, J. P. (2002). The Dynamics of Self-Esteem : A Growth-Curve Analysis. Journal of Youth and Adolescence, vol. 31, $\mathrm{n}^{\mathrm{0}} 2$, p. 101-113.

BEAUCHESNE, C., et DUMAS, S. (1993). Étudier et travailler. Enquête réalisée auprès des jeunes du secondaire sur le travail rémunéré durant l'année scolaire. Québec: Ministère de l'Éducation, Gouvernement du Québec, p. 1-31.

BEN-ZUR, H. (2003). Happy Adolescents : The Link between Subjective Well-Being, Internal Resources, and Parental Factors. Journal of Youth and Adolescence, vol. 32, no 2, p. 67-79.

BOBO, J. K., JANET K., GILCHRIST, L.-D., ELMER, J. F., SNOW, W. H. et SCHINKE, S. P. (1986). Hassles, Role Strain, and Peer Relations in Young Adolescents. Journal of Early Adolescence, vol. 6, nº 4, p. 339-352.

COMMISSION DES NORMES DU TRAVAIL (octobre 2003). Les normes du travail au Québec. Québec: Gouvernement du Québec.

CREED, P. A., PATTON, W. et BARTRUM, D. (février 2002). Multidimensional Properties of the LOT-R : Effects of Optimism and Pessimism on Career and Well-Being Related Variables in Adolescents. Journal of Career Assessment, vol. $10, \mathrm{n}^{\mathrm{0}} 1$, p. $42-61$.

D’AMICO, R. (juillet 1984). Does Employment during High School Impair Academic Progress? Sociology of Education, vol. 57, p. 152-164.

DESLANDES, R., POTVIN, P. et LECLERC, D. (1999). Validation québécoise de l'échelle de l'autonomie de l'adolescent. Science et Comportement, vol. 27, $\mathrm{n}^{\mathrm{o}} 1, \mathrm{p} .37-51$.

DUBOIS, D. L., BULL, C. A., SHERMAN, M. D. et ROBERTS, M. (1998). Self-Esteem and Adjustment in Early Adolescence: A Social-Contextual Perspective. Journal of Youth and Adolescence, vol. 27, $\mathrm{n}^{0}$ 5, p. 557-583.

DUMONT, M. (mars-avril 2005). Impact du travail à temps partiel sur l'adaptation psychosociale et scolaire des adolescents. Bulletin du CRIRES. Nouvelles CSQ, p. 1-4. 
DUMONT, M. (2000). Expérience du stress à l'adolescence. Journal international de psychologie, vol 35, n5, p. 194-206.

DUMONT, M., LECLERC, D. et DESLANDES, R. (2003). Ressources personnelles et détresse psychologique en lien avec le rendement scolaire et le stress chez des élèves de quatrième secondaire. Revue canadienne des sciences $d u$ comportement, vol 35, n 3, p. 254-267.

DUMONT, M., LECLERC, D., MASSÉ, L. et MCKINNON, S. (du 7 au 10 juillet 2006). Conciliation études - travail chez les adolescents. Affiche présentée au $14^{\mathrm{e}}$ congrès de l'AIPTLF (l'Association Internationale de Psychologie du Travail de langue Française), Hammamet, Tunisie.

DUMONT, M. et PROVOST, M. (1999). Resilience in Adolescents : The Protective Role of Social Support, Coping Strategies, Self-Esteem, and Social Activities on Experience of Stress and Depression. Journal of Youth and Adolescence, vol. 28, $n^{0} 3$, p. 343-363.

DUMONT, M., LECLERC, D. et PRONOVOST, J. (2000). Profils psychopathologiques et stratégies adaptatives chez des adolescents. Revue européenne de psychologie appliquée, vol. 50, no 1, p. 59-69.

FRYDENBERG, E. et LEWIS, R. (1993). Measuring the Concerns of Australian Adolescents : Developping a Concise Classificatory System. Australian Educational Researcher, vol. 23, $\mathrm{n}^{0}$ 1, p. 47-64.

GREEN, D. L. (1990). High School Student Employment in Social Context : Adolescents' Perceptions of the Role of Part-Time Work. Adolescence, vol. XXV, $\mathrm{n}^{0}$ 98, p. 425-434.

GREENBERGER, E., JOSSELSON, R., KNERR, C. et KNERR, B. (1974). The Measurement and Structure of Psychosocial Maturity. Journal of Youth and Adolescence, vol. 4, p. 127-143.

GREENGLASS, E.R. (2002). Proactive Coping and Quality of Life Management, dans Beyond Coping Meeting Goals, Visions, and Challenges, sous la direction de Erica Frydenberg. New York: Oxford University Press, p. 37-62.

GRIFFITH, M. A., DUBOW, E. F. et IPPOLITO, M. F. (2000). Developmental and Cross-Situational Differences in Adolescents' Coping Strategies. Journal of Youth and Adolescence, vol. 29, $\mathrm{n}^{\circ}$ 2, p. 183-204.

GUYON, L. et DESJARDINS, L. (2002). Consommation d'alcool et de drogues chez les élèves du secondaire au Québec en 2002, dans L'alcool, les drogues, le jeu: les jeunes sont-ils preneurs?, sous la direction de l'Institut de la statistique du Québec. Québec: Gouvernement du Québec, p. 35-65.

KOUVONEN, A. et LINTONEN, T. (2002a). Adolescent Work and Drug Experiments. Journal of Substance Use, vol. 7, p. 85-92.

KOUVONEN, A. et LINTONEN, T. (2002b). Adolescent Part-Time Work and Heavy Drinking in Finland. Addiction, vol. 97, p. 311-318. 
LARGIE, S., FIELD, T., HERNANDEZ-REIF, M., SANDERS, C. E. et DIEGO, M. (2001). Employment During Adolescence is Associated With Depression, Inferior Relationships, Lower Grades, and Smoking. Adolescence, vol. 36, $\mathrm{n}^{\circ}$ 142, p. 395-401.

LECLER, D., PRONOVOST, J. et DUMONT, M. (2004). Psychological Symptoms, Suicidal Risk and Coping on Adolescents. Poster-discussion presented to the 9th Biennal Conference of the European Association for Research on Adolescence, EARA, Porto, Portugal, May 5-8.

MINISTÈRE DE L'ÉDUCATION DU QUÉBEC (1994). La consommation de drogues licites et illicites chez les filles et les garçons du secondaire et les conduites suicidaires (1994). Québec: Gouvernement du Québec, 115 p.

MINISTÈRE DU TRAVAIL (1998). Document de réflexion sur le travail des enfants au Québec. Québec : Ministère du Travail, pagination multiple.

MORTIMER, J. T., HARLEY, C. et STAFF, J. (2002). The Quality of Work and Youth Mental Health. Work and Occupations, vol. 29, nº 2, p. 166-197.

MORTIMER, J. T. et STAFF, J. (2004). Early Work as a Source of Developmental Discontinuity during the Transition to Adulthood. Development and Psychopathology, vol. 16, no 4, p. 1047-1070.

PLANCHEREL, B., BETTSCHART, W., BOLOGNINI, M., DUMONT, M. et HALFON, O. (1997). Influence comparée des événements existentiels et des tracas quotidiens sur la santé psychique à la préadolescence. Revue de neuropsychiatrie de l'enfance et de l'adolescence, vol. 45, nº 3, p. 126-138.

PRONOVOST, J., LECLERC, D. et DUMONT, M. (2003). Facteurs de protection reliés au risque suicidaire chez des adolescents : Comparaison de jeunes du milieu scolaire et de jeunes en centres jeunesse. Revue québécoise de psychologie, vol. 24, no 1, p. 179-199.

REYNOLDS, L. K., O'KOON, J. H., PAPADEMETRIOU, E., SZCZYGIEL S. et GRANT, K. E. (2001). Stress and Somatic Complaints in Low-Income Urban Adolescents. Journal of Youth and Adolescence, vol. 30, no 4, p. 499-514.

ROSENBERG, M. (1965). Society and the Adolescent Self-Image. New York: Princeton University Press, 347 p.

SAFRON, D. J., SCHULENBERG, J. E. et BACHMAN, J. G. (décembre 2001). Part-Time Work and Hurried Adolescence : The Links Among Work Intensity, Social Activities, Health Behaviors, and Substance Use. Journal of Health and Social Behavior, vol. 42, p. 425-449.

SANTANA, V. S., COOPER, S. P., ROBERTS, R. E. et ARAÚJO-FILHO, J. B. (2005). Adolescent Students Who Work: Gender Differences in School Performances and Self-Perceived Health. Int. Journal Environ. Health, vol. 11, no 3 , p. 294-301. 
SCHEIER, M. F. et CARVER, C. S. (1985). Optimism, Coping, and Health : Assessment and Implications of Generalized Outcome Expectancies. Health Psychology, vol. 4, p. 219-247.

SCHEIER, M. F., KUMARI-WEINTRAUB, J. et CARVER, C. S. (1986). Coping with Stress : Divergent Strategies of Optimists and Pessimists. Journal of Personality and Social Psychology, vol. 51, $\mathrm{n}^{\mathrm{0}}$ 6, p. 1257-1264.

SCHMEELK-CONE, K. H. et ZIMMERMAN, M. A. (2003). A Longitudinal Analysis of Stress in African American Youth : Predictors and Outcomes of Stress Trajectories. Journal of Youth and Adolescence, vol. 32 (6), p. 419-430.

SEIFFGE-KRENKE, I. (2000). Causal Links between Stressful Events, Coping Style, and Adolescent Symptomatology. Journal of Adolescence, vol. 23, p. 675-691.

STEINBERG, L. et DORNBUSCH, S. M. (1991). Negative Correlates of Part-Time Employment during Adolescence : Replication and Elaboration. Developmental Psychology, vol. 27, no 2, p. 304-313.

STEINHAUSEN, H.-C. et WINKLER METZKE, C. (2001). Risk, Compensatory, Vulnerability, and Protective Factors Influencing Mental Health in Adolescence. Journal of Youth and Adolescence, vol. 30, nº 3, p. 259-280. 